\title{
Cystic fibrosis, primary ciliary dyskinesia and non-cystic fibrosis bronchiectasis: update 2008-11
}

\author{
W G Flight, ${ }^{1,2}$ A M Jones ${ }^{1,2}$
}

${ }^{1}$ Manchester Adult Cystic Fibrosis Centre, South Manchester University Hospitals NHS Trust, Manchester, UK ${ }^{2}$ Respiratory Research Group, University of Manchester, UK

\section{Correspondence to}

Dr A M Jones, Manchester Adult Cystic Fibrosis Centre, South Manchester University Hospitals NHS Trust, Southmoor Road, Wythenshawe, Manchester M23 9LT, UK; andrew.jones@uhsm.nhs.uk

WGF and AMJ contributed equally to the manuscript.

Received 13 May 2011 Accepted 14 May 2011 Published Online First 15 June 2011

\begin{abstract}
A review is presented of key clinical papers published in Thorax and elsewhere between 2008 and April 2011 which have advanced our understanding of cystic fibrosis (CF), primary ciliary dyskinesia and non-CF

bronchiectasis. Studies were identified through searches of the Thorax archive and the Medline database. Within the field of CF, the following key themes were studied: diagnosis in equivocal CF, assessment of CF lung disease, novel therapies addressing the basic defect in $\mathrm{CF}$, maintenance pulmonary therapies, management of early Pseudomonas infection, the microbiology of CF lung disease, renal impairment in $\mathrm{CF}$ and controversies in lung transplantation in CF.
\end{abstract}

\section{INTRODUCTION}

Over the last 3 years there have been significant advances in our understanding of chronic suppurative lung diseases. In this article we review a selection of key papers published in Thorax on the topics of cystic fibrosis (CF), primary ciliary dyskinesia (PCD) and non-CF bronchiectasis between 2008 and 2011 and set them in the context of the wider respiratory literature. While endeavouring to be comprehensive, we have elected to focus on clinical studies with practical implications for the diagnosis, management and prognosis of patients with these conditions.

\section{NON-CF BRONCHIECTASIS}

As can be seen from a glance at this review or a simple medical database search, the volume of work published on non-CF bronchiectasis and PCD is dwarfed by the research output on CF. The large gaps in the non-CF bronchiectasis evidence base were highlighted in the 2010 BTS bronchiectasis guidelines ${ }^{1}$ and are of ongoing concern. The arrival of a relatively large randomised controlled trial of nebulised gentamicin in patients with non-CF bronchiectasis is therefore highly welcome. ${ }^{2}$ Murray and colleagues from Edinburgh randomised 65 patients to either nebulised gentamicin $80 \mathrm{mg}$ twice daily or placebo for a period of 12 months. Although no improvement in lung function was seen, the gentamicin group had substantial improvements in exercise capacity and quality of life scores alongside an impressive doubling of the time to next exacerbation (120 vs 61.5 days). Dhar et al have reported their experiences with nebulised colistin in a small cohort of patients with non-CF bronchiectasis infected with Pseudomonas aeruginosa. ${ }^{3}$ Regular colistin appeared to reduce the frequency of exacerbations and hospitalisation, a finding which now needs confirmation in larger controlled trials.
The prognosis for children with non-CF bronchiectasis has become clearer in recent years through the publication of three observational studies. Bastardo et al provided longitudinal data on lung function and growth in children predominantly with either idiopathic bronchiectasis or primary immunodeficiency. ${ }^{4}$ Their cohort of 59 UK patients with a median age of 8.2 years had normal height, weight and body mass index at recruitment. Growth was satisfactory over the 3-5-year followup period and spirometric values remained stable during the study; neither a fall in lung function suggesting progressive disease nor an improvement suggesting response to treatment was seen. Kapur and colleagues from Australia subsequently produced data broadly in agreement with these findings, suggesting that lung function remains stable in patients with bronchiectasis managed in a tertiary centre. ${ }^{5}$ Conversely, a group from New Zealand previously reported in this journal that children with non-CF bronchiectasis lost $1.9 \%$ of their best forced expiratory volume in $1 \mathrm{~s}\left(\mathrm{FEV}_{1}\right)$ per year over a mean follow-up period of 5.7 years. ${ }^{6}$ The explanation for the differences between these three cohorts remains unclear, but is likely to reflect a combination of differing ethnic backgrounds, aetiologies, healthcare systems and severity at baseline.

\section{PRIMARY CILIARY DYSKINESIA}

PCD refers to a group of autosomal recessive conditions causing impaired function of the respiratory epithelial cilia. The clinical effects of PCD include bronchiectasis, male infertility and recurrent sinusitis. ${ }^{8}$ Marthin and colleagues from the National Danish PCD Centre published a study in 2010 representing an important milestone in our understanding of the natural history of PCD. ${ }^{9}$ In a cohort of 74 patients there was considerable variability in the course of lung function over several decades. The potential for a substantial decline in lung function, seen in $10 \%$ of patients, is an important finding which highlights the need for accurate diagnosis and specialist follow-up of these patients.

Standard diagnostic techniques, as recommended in a European consensus statement, utilise electron microscopy and high-speed ciliary motion analysis of nasal epithelial biopsy samples. ${ }^{8}$ In a proportion of patients, however, these tests prove inconclusive. Evidence has accumulated to suggest that culture of cilia from nasal brushing samples may help to clarify the diagnosis of PCD. Pifferi et al found that the combination of electron microscopy, ciliary motion analysis and cell culture provided a firm diagnosis or exclusion of PCD in $56 / 59$ cases 
$(95 \%) .{ }^{10}$ In those with equivocal standard tests, the addition of cell culture allowed a firm diagnosis in two-thirds of cases (6/9). Hirst et al used an alternative air-liquid interface system to culture ciliated cells with a success rate of $54 \%$ leading to the exclusion of PCD in eight patients. ${ }^{11}$ Where the culture was successful, this group was able to go on and perform electron microscopy of the cultured cilia for the first time. The precise role of these techniques is likely to evolve over the coming years and expand to encompass genetic analysis in PCD, thereby enhancing our diagnostic abilities. ${ }^{8}$

\section{CYSTIC FIBROSIS}

\section{Diagnostic uncertainty in CF: evolving strategies}

Providing an accurate diagnosis in patients with atypical presentations or equivocal sweat test results is a considerable challenge for CF physicians. Despite the production of consensus guidelines on the diagnosis of CF, there is still no 'gold standard' test. ${ }^{12} 13$ Great interest has been shown in recent years in the development of alternative methods of assessing CFTR function with the aim of improved diagnostic clarity.

Sermet-Gaudelus et al used nasal potential difference (NPD) measurement to investigate a group of 23 children with positive newborn screening tests but indeterminate sweat tests. ${ }^{14}$ All had fewer than two CFTR mutations identified by genetic analysis. The authors identified thresholds for a CF range by comparison with both healthy controls and a group with classic CF. Thirteen patients had NPD results within the 'CF range' and, at 3 years of follow-up, 69\% of these had developed evidence of chronic sinoplumonary disease compared with none of those classified as being in the 'control' range. In a separate study the investigators also investigated NPD in older children with suspected CF and equivocal sweat tests. ${ }^{15}$ Over half the patients with abnormal NPD had two CFTR mutations compared with $14 \%$ of those with normal NPD, and $86 \%$ had phenotypic features of CF such as recurrent respiratory tract infections. A key observation is that this group was able to perform measurements in children as young as 3 months where previously this was thought to be a major limitation of NPD. ${ }^{12}$

Alternative techniques to NPD for the characterisation of CFTR function include intestinal current measurement ${ }^{16}$ and the more recently described skin sweat gland electrical measurement. ${ }^{17}$ Both have shown promise as an aid to the diagnosis of equivocal CF, but head-to-head studies comparing these techniques with the more widely established NPD method are required.

Alongside refinement of these diagnostic techniques there is a need for a greater understanding of the natural history of patients with CFTR dysfunction. Retrospective pan-European data reported by Goubau et al in Thorax in 2009 gives some insight into this. ${ }^{18}$ Patients with CFTR dysfunction (defined as sweat chloride $30-60 \mathrm{mmol} / \mathrm{l}$ and two CFTR mutations or abnormal NPD) had evidence of respiratory disease with an increased incidence of respiratory infections and isolation of $P$ aeruginosa or Staphylococcus aureus compared with those classified as 'CF unlikely' (ie, intermediate sweat test but $<2$ CFTR mutations or normal NPD). The authors conclude that CFTR dysfunction is a distinct phenotype somewhere between pancreatic-sufficient CF and asymptomatic carrier status.

\section{ASSESSMENT AND MONITORING OF CF LUNG DISEASE}

$\mathrm{FEV}_{1}$ remains the 'gold standard' means of assessing the progression of lung disease in CF but it has several limitations. As life expectancy in CF increases so the rate of decline of $\mathrm{FEV}_{1}$ has slowed, limiting its suitability as a primary outcome measure for all but very large or long-term research studies. $\mathrm{FEV}_{1}$ is also insensitive to the identification of early lung disease where the predominant pathology is in the small airways. The lung clearance index (LCI) is derived from a multiple breath washout technique and has been proposed as an alternative outcome measure in CF clinical studies. ${ }^{19} 20$ The LCI value rises with increasing heterogeneity of ventilation and thus with increased disease severity. An attractive feature of LCI is that it is performed during tidal breathing and has been shown to be tolerated well by young children. ${ }^{21}$

Horsley and colleagues from the UK CF Gene Therapy Consortium evolved a means of measuring LCI using technology originally designed for the non-invasive measurement of cardiac output. $^{22}$ LCI rose in proportion to falling $\mathrm{FEV}_{1}$ but was significantly more sensitive in identifying early CF lung disease in adults. Complementary findings in paediatric patients have been reported by Gustafsson et $a^{23}$ and Owens et $a^{24}$ who report a strong correlation between LCI and high-resolution CT (HRCT) abnormalities. LCI at the age of 4 years also appeared to be predictive of abnormal spirometry at 6-10 years of age in a cohort followed by the London CF Collaboration. ${ }^{25}$ Those patients with normal $\mathrm{FEV}_{1}$ but abnormal LCI may represent a target for early intervention to prevent disease progression. For instance, Amin et al used LCI to assess response to therapy with inhaled $7 \%$ hypertonic saline. ${ }^{26}$ In a randomised crossover trial involving 20 patients, hypertonic saline led to a significant improvement in LCI after 4 weeks compared with nebulised normal saline. There was no difference between the two groups in $\mathrm{FEV}_{1}$, forced vital capacity or forced expiratory flow at $25-75 \%$ of FVC.

The role of thoracic HRCT scans in the assessment of CF lung disease continues to be debated. Work by Sly et al has shown that over $80 \%$ of children diagnosed with CF through newborn screening have abnormal HRCT scans at just 3 months of age. ${ }^{27}$ Various CT severity scores and protocols have been described, although no consensus has been reached and it remains unclear as to the utility of CT scans for long-term monitoring of $\mathrm{CF}^{28-30}$

\section{AIRWAY CLEARANCE AND RESPIRATORY SUPPORT}

One notable difference in CF care between North America and Europe is the marked discrepancy in the use of high-frequency chest wall oscillation (HFCWO) vests as an aid to sputum expectoration. HFCWO is considered standard therapy in the USA. Osman et al reported in 2010 a randomised crossover trial of HFCWO versus patients' usual airway clearance technique (ACT) over a 4-day period. ${ }^{31}$ ACT led to a significantly higher sputum weight than HFCWO, and ACT was preferred by just over half of the participants. In an accompanying editorial, Bradley has emphasised the need for larger long-term comparative studies of the various ACT strategies. ${ }^{32}$

Inhaled therapies to enhance sputum clearance in CF are a key part of maintenance CF care. Mannitol is an osmotic agent that has recently been evaluated as an inhaled therapy in CF. The theoretical mechanism of action of mannitol is through increased hydration of the airway surface liquid allowing easier expectoration of sputum. Two recent studies have evaluated the potential use of inhaled mannitol in CF. Minasian et al reported a modest improvement in $\mathrm{FEV}_{1}$ in children with $\mathrm{CF}$ given 12 weeks of inhaled mannitol, ${ }^{33}$ while Bilton and colleagues have recently reported the results of a large international phase III study of inhaled mannitol in CF. In this study, mannitol led to a modest improvement in absolute $\mathrm{FEV}_{1}$ and a reduction in the number of exacerbations for those patients able to continue 
with the therapy. ${ }^{34}$ The tolerability of inhaled mannitol may well limit its widespread use.

For over two decades non-invasive ventilation (NIV) has been used as a 'bridge to lung transplant', but evidence for substantial long-term clinical benefits in advanced CF remains elusive. Young et al performed a randomised crossover trial of nocturnal NIV, oxygen and supplemental air in eight patients with CF and hypercapnia. ${ }^{35}$ NIV led to improvements in respiratory symptoms, shuttle walk distances and nocturnal transcutaneous carbon dioxide measurements but failed to impact on daytime carbon dioxide tension. Further studies are required to establish the precise indications and optimal timing for institution of long-term NIV in CF.

\section{MICROBIOLOGY OF CF LUNG DISEASE}

Chronic infection with $P$ aeruginosa is associated with reduced survival, worsening lung function and increased morbidity. Strategies to reduce the incidence of chronic $P$ aeruginosa infection are key components of CF care.

The use of serological testing to detect antibodies to $P$ aeruginosa has been used extensively in paediatric practice where many patients are unable to produce sputum regularly. Douglas and colleagues compared the diagnostic value of serology with the culture of bronchoalveolar lavage fluid as part of two separate observational studies in young children. ${ }^{36}$ In a combined population of 131 patients, serology had an extremely low positive predictive value of between 0.23 and 0.43 , rendering it unsuitable for the diagnosis of $P$ aeruginosa infection. A high negative predictive value of $\geq 0.94$ was seen, however, suggesting that serology may still have a role as a screening test for $P$ aeruginosa.

A potential source of pathogens such as $P$ aeruginosa in CF is the patients' own upper airways. Mainz et al performed paired sputum cultures and nasal lavage in 182 patients. ${ }^{37}$ Nasal lavage identified $P$ aeruginosa in $55.6 \%$ of cases of known chronic infection. Genetic analysis confirmed the presence of identical strains in both upper and lower airways in $96 \%$ of cases with positive paired cultures. There may be a future role for upper airway sampling as a surrogate marker of lower airway infection in non-expectorating patients. The full ramifications of the upper airway as a reservoir and potential source of acquisition of CF pathogens need further exploration.

There is compelling evidence that certain $P$ aeruginosa strains are transmitted between patients with CF. Wainwright and colleagues recently demonstrated that $P$ aeruginosa-as well as Burkholderia cenocepacia, Achromobacter xylosoxidans and Stenotrophomonas maltophilia-could all be cultured from samples collected using a cough aerosol sampling system. ${ }^{38}$ Twenty-five of 28 samples from patients with known chronic $P$ aeruginosa infection produced positive cough aerosols, and in four of these cases air sampling of the clinic room was also positive. The study strongly suggests that the aerosol route is one potential mode of transmission for these pathogens. ${ }^{39}$

Two recent studies have provided valuable information to guide therapeutic strategies to prevent chronic $P$ aeruginosa infection. Ratjen et al conducted a randomised study comparing either 28 or 56 days of nebulised tobramycin $300 \mathrm{mg}$ twice daily. ${ }^{40}$ Participants had been free of $P$ aeruginosa for at least 1 year with a minimum of four respiratory samples taken over this period. Approximately one-third of patients in each group re-isolated $P$ aeruginosa over the study follow-up period. The median time to recurrence of $P$ aeruginosa was 26 months in both the 28- and 56-day treatment groups. Both regimens were tolerated well with a low incidence of adverse events. Given these findings, extending inhaled tobramycin to 56 days for $P$ aeruginosa eradication appears unnecessary. ${ }^{41}$

Tramper-Stranders et al investigated an alternative approach-namely, the use of regular prophylactic courses of anti-Pseudomonal antibiotics to try and prevent chronic infection. ${ }^{42} \mathrm{~A} 3$-week course of inhaled colistin and oral ciprofloxacin was given every 3 months to one group of children with CF while a control group received an inhaled and oral placebo. The primary outcome measure was identification of $P$ aeruginosa in two respiratory cultures at least 1 week apart. No difference between the groups was seen in rates of Paeruginosa isolation or lung function, suggesting that the cycled antibiotic approach offers no direct advantage over culture-directed therapy. ${ }^{43}$

Kolpen and colleagues have shown that polymorphonuclear leucocytes consume oxygen in the sputum of patients with chronic $P$ aeruginosa infection, ${ }^{44}$ and steep oxygen gradients exist within CF airways with regions of hypoxia within mucus plugs. Interest has therefore been growing in a potential role for anaerobic bacteria in CF lung disease. Tunney et al recently identified anaerobes in the sputum of all 26 patients commencing antibiotics for an exacerbation of $\mathrm{CF}^{45}$ In vitro data from Ulrich et al have suggested that the anaerobe Prevotella intermedia is cytotoxic to respiratory epithelial cells, suggesting a possible pathogenic role, ${ }^{46}$ although the clinical extent of this is yet to be elucidated. ${ }^{47}$

\section{ACUTE RENAL FAILURE IN CF}

Patients with CF are at risk of renal injury: frequent use of nephrotoxic drugs, concurrent CF-related diabetes mellitus and dehydration as a result of gastrointestinal complications of CF may all contribute. ${ }^{48}$ Smyth et al conducted a case-control study to investigate paediatric patients who had suffered an episode of acute renal failure (ARF). ${ }^{49}$ Exposure to intravenous aminoglycosides was strongly linked to ARF with a staggering OR of 81.8 (95\% CI 4.7 to 1427). The risk of ARF seemed to be particularly strong with gentamicin use (19/24 cases), but only $1 / 42$ controls had received gentamicin over the previous year. The authors consequently recommend the use of tobramycin as the intravenous aminoglycoside of choice in CF. Strategies to further reduce the risk of renal impairment due to aminoglycosides are required. ${ }^{50}$

\section{CF AND LUNG TRANSPLANTATION}

Despite ongoing improvements in survival for patients with CF, respiratory failure due to chronic endobronchial sepsis almost invariably occurs. For those deemed to be 'good candidates', lung transplantation is the final remaining treatment option in this situation. The suitability for transplantation of those patients chronically infected with Burkholderia cepacia complex (BCC) has long been questioned, ${ }^{51}$ and important evidence to add to this debate has recently been published.

The Newcastle transplant centre in the UK reported its outcomes in all 176 patients with CF receiving lung transplants over a period of two decades. ${ }^{52}$ Survival rates at 1,5 and 10 years in this cohort were $82 \%, 62 \%$ and $51 \%$, respectively. Data were subsequently presented by the authors with specific regard to outcomes in patients chronically infected with BCC. ${ }^{53}$ Twentytwo of 216 cases were infected with BCC before transplantation, 12 of whom had $B$ cenocepacia infection. The $B$ cenocepacia group had a mortality rate at 1 year of $75 \%$, with eight out of nine patients dying of sepsis and BCC bacteraemia.

Findings by Boussaud et al broadly concur with the Newcastle experience of CF patients infected with BCC. ${ }^{54} \mathrm{~A}$ similar 
number of patients in this study (22/247) were infected with $\mathrm{BCC}$ before transplantation. Median survival in the non-BCC group was 79 months compared with 43 months with BCC although, given the small numbers, this did not reach statistical significance. A significantly higher risk of death was seen with some but not all strains of $B$ cenocepacia. Murray et al reported outcomes in 88 BCC-infected patients identified through the US Cystic Fibrosis Foundation registry. ${ }^{55}$ Post-transplant mortality was increased in those harbouring $B$ cenocepacia and Burkholderia gladioli, although patients infected with Burkholderia multivorans had similar survival rates to uninfected patients.

Going forward, there are ongoing variations between groups in acceptance for transplantation of patients infected with Burkholderia species. The Newcastle unit has concluded that the risk of transplantation in patients with $B$ cenocepacia is too great, whereas the French group stop short of precluding these patients all together. It is likely that policy in this ethically challenging area will continue to excite controversy. ${ }^{56}$

\section{SUMMARY}

Over the last 3 years the respiratory research community has provided a wealth of vital clinical evidence in the field of CF and other suppurative lung conditions. The forward strides in the management of CF are sure to accelerate with the advent of CFTR-targeted therapies. ${ }^{57} 58$ Over the next 3 years we also hope to see a corresponding increase in our understanding of the diverse entity that is non-CF bronchiectasis.

Competing interests None.

Provenance and peer review Not commissioned; internally peer reviewed

\section{REFERENCES}

1. Pasteur MC, Bilton D, Hill AT; British Thoracic Society Bronchiectasis non CFGG. British Thoracic Society guideline for non-CF bronchiectasis. Thorax 2010:65(Suppl 1):i1-58.

2. Murray MP, Govan JR, Doherty CJ, et al. A randomized controlled trial of nebulized gentamicin in non-cystic fibrosis bronchiectasis. Am J Respir Crit Care Med 2011:183:491-9.

3. Dhar R, Anwar GA, Bourke SC, et al. Efficacy of nebulised colomycin in patients with non-cystic fibrosis bronchiectasis colonised with Pseudomonas aeruginosa. Thorax 2010;65:553

4. Bastardo CM, Sonnappa S, Stanojevic S, et al. Non-cystic fibrosis bronchiectasis in childhood: longitudinal growth and lung function. Thorax 2009:64:246-51.

5. Kapur N, Masters IB, Chang AB. Longitudinal growth and lung function in pediatric non-cystic fibrosis bronchiectasis: what influences lung function stability? Chest 2010:138:158-64.

6. Twiss J, Stewart AW, Byrnes CA. Longitudinal pulmonary function of childhood bronchiectasis and comparison with cystic fibrosis. Thorax 2006:61:414-18.

7. Loebinger MR, Bilton D, Wilson R. Upper airway 2: Bronchiectasis, cystic fibrosis and sinusitis. Thorax 2009;64:1096-101.

8. Barbato A, Frischer T, Kuehni CE, et al. Primary ciliary dyskinesia: a consensus statement on diagnostic and treatment approaches in children. Eur Respir $J$ 2009:34:1264-76.

9. Marthin JK, Petersen N, Skovgaard LT, et al. Lung function in patients with primary ciliary dyskinesia: a cross-sectional and 3-decade longitudinal study. Am J Respir Crit Care Med 2010;181:1262-8.

10. Pifferi M, Montemurro F, Cangiotti AM, et al. Simplified cell culture method for the diagnosis of atypical primary ciliary dyskinesia. Thorax 2009;64:1077-81.

11. Hirst RA, Rutman A, Williams $G$, et al. Ciliated air-liquid cultures as an aid to diagnostic testing of primary ciliary dyskinesia. Chest 2010;138:1441-7.

12. Farrell PM, Rosenstein BJ, White TB, et al. Guidelines for diagnosis of cystic fibrosis in newborns through older adults: Cystic Fibrosis Foundation consensus report. J Pediatr 2008;153:S4-S14.

13. De Boeck K, Wilschanski M, Castellani C, et al. Cystic fibrosis: terminology and diagnostic algorithms. Thorax 2006;61:627-35.

14. Sermet-Gaudelus I, Girodon E, Roussel D, et al. Measurement of nasal potential difference in young children with an equivocal sweat test following newborn screening for cystic fibrosis. Thorax 2010;65:539-44.

15. Sermet-Gaudelus I, Girodon E, Sands D, et al. Clinical phenotype and genotype of children with borderline sweat test and abnormal nasal epithelial chloride transport. Am J Respir Crit Care Med 2010;182:929-36.
16. Derichs N, Sanz J, Von Kanel T, et al. Intestinal current measurement for diagnostic classification of patients with questionable cystic fibrosis: validation and reference data. Thorax 2010;65:594-9.

17. Gonska T, Ip W, Turner D, et al. Sweat gland bioelectrics differ in cystic fibrosis a new concept for potential diagnosis and assessment of CFTR function in cystic fibrosis. Thorax 2009:64:932-8.

18. Goubau C, Wilschanski M, Skalicka V, et al. Phenotypic characterisation of patients with intermediate sweat chloride values: towards validation of the European diagnostic algorithm for cystic fibrosis. Thorax 2009:64:683-91.

19. Davies JC, Cunningham S, Alton EW, et al. Lung clearance index in CF: a sensitive marker of lung disease severity. Thorax 2008;63:96-7.

20. Aurora P. Multiple-breath inert gas washout test and early cystic fibrosis lung disease. Thorax 2010;65:373-4.

21. Aurora P, Bush A, Gustafsson P, et al. Multiple-breath washout as a marker of lung disease in preschool children with cystic fibrosis. Am J Respir Crit Care Med 2005; 171:249-56.

22. Horsley AR, Gustafsson PM, Macleod KA, et al. Lung clearance index is a sensitive repeatable and practical measure of airways disease in adults with cystic fibrosis. Thorax 2008;63:135-40

23. Gustafsson PM, De Jong PA, Tiddens HA, et al. Multiple-breath inert gas washout and spirometry versus structural lung disease in cystic fibrosis. Thorax 2008:63:129-34

24. Owens CM, Aurora P, Stanojevic S, et al. Lung Clearance Index and HRCT are complementary markers of lung abnormalities in young children with CF. Thorax 2011;66:481-8.

25. Aurora P, Stanojevic S, Wade A, et al. Lung clearance index at 4 years predicts subsequent lung function in children with cystic fibrosis. Am J Respir Crit Care Med 2011:183:752-8

26. Amin R, Subbarao P, Jabar A, et al. Hypertonic saline improves the $\mathrm{LCl}$ in paediatric patients with CF with normal lung function. Thorax 2010;65:379-83.

27. Sly PD, Brennan S, Gangell C, et al. Lung disease at diagnosis in infants with cystic fibrosis detected by newborn screening. Am J Respir Crit Care Med 2009:180:146-52.

28. Loeve $\mathbf{M}$, Lequin $\mathbf{M H}$, de Bruijne $\mathbf{M}$, et al. Cystic fibrosis: are volumetric ultra-lowdose expiratory CT scans sufficient for monitoring related lung disease? Radiology 2009:253:223-9.

29. Loeve $\mathbf{M}$, van Hal PT, Robinson $\mathrm{P}$, et al. The spectrum of structural abnormalities on CT scans from patients with CF with severe advanced lung disease. Thorax 2009:64:876-82

30. Cademartiri F, Luccichenti G, Palumbo AA, et al. Predictive value of chest CT in patients with cystic fibrosis: a single-center 10-year experience. AJR Am J Roentgenol 2008;190:1475-80.

31. Osman LP, Roughton M, Hodson ME, et al. Short-term comparative study of high frequency chest wall oscillation and European airway clearance techniques in patients with cystic fibrosis. Thorax 2010;65:196-200.

32. Bradley JM. High frequency chest wall oscillation in cystic fibrosis. Thorax 2010;65:189-90.

33. Minasian C, Wallis C, Metcalfe C, et al. Comparison of inhaled mannitol, daily rhDNase and a combination of both in children with cystic fibrosis: a randomised trial. Thorax 2010:65:51-6.

34. Bilton D, Robinson $P$, Cooper $P$, et al. Inhaled dry powder mannitol in cystic fibrosis: an efficacy and safety study. Eur Respir J 2011;38:1071-80.

35. Young AC, Wilson JW, Kotsimbos TC, et al. Randomised placebo controlled trial of non-invasive ventilation for hypercapnia in cystic fibrosis. Thorax 2008;63:72-7.

36. Douglas TA, Brennan S, Berry L, et al. Value of serology in predicting Pseudomonas aeruginosa infection in young children with cystic fibrosis. Thorax 2010;65:985-90

37. Mainz JG, Naehrlich L, Schien M, et al. Concordant genotype of upper and lower airways $\mathrm{P}$ aeruginosa and $\mathrm{S}$ aureus isolates in cystic fibrosis. Thorax 2009;64:535-40.

38. Wainwright CE, France MW, O'Rourke $\mathrm{P}$, et al. Cough-generated aerosols of Pseudomonas aeruginosa and other Gram-negative bacteria from patients with cystic fibrosis. Thorax 2009;64:926-31.

39. Saiman L. Furthering our understanding of pathogen transmission in cystic fibrosis Thorax 2009;64:921-2.

40. Ratjen F, Munck A, Kho $P$, et al. Treatment of early Pseudomonas aeruginosa infection in patients with cystic fibrosis: the ELITE trial. Thorax 2010;65:286-91

41. Smyth AR. Pseudomonas eradication in cystic fibrosis: who will join the ELITE? Thorax 2010;65:281-2

42. Tramper-Stranders GA, Wolfs TF, van Haren Noman S, et al. Controlled trial of cycled antibiotic prophylaxis to prevent initial Pseudomonas aeruginosa infection in children with cystic fibrosis. Thorax 2010;65:915-20.

43. Eber $\mathbf{E}$, Zach MS. Pseudomonas aeruginosa infection in cystic fibrosis: prevent, eradicate or both? Thorax 2010:65:849-51.

44. Kolpen M, Hansen CR, Bjarnsholt T, et al. Polymorphonuclear leucocytes consume oxygen in sputum from chronic Pseudomonas aeruginosa pneumonia in cystic fibrosis. Thorax 2010:65:57-62.

45. Tunney MM, Klem ER, Fodor AA, et al. Use of culture and molecular analysis to determine the effect of antibiotic treatment on microbial community diversity and abundance during exacerbation in patients with cystic fibrosis. Thorax. Published Online First: 26 January 2011. doi:10.1136/thx.2010.137281. 
46. Ulrich M, Beer I, Braitmaier P, et al. Relative contribution of Prevotella intermedia and Pseudomonas aeruginosa to lung pathology in airways of patients with cystic fibrosis. Thorax 2010;65:978-84.

47. Jones AM. Anaerobic bacteria in cystic fibrosis: pathogens or harmless commensals? Thorax 2011;66:558-9.

48. Prayle A, Watson A, Fortnum $\mathrm{H}$, et al. Side effects of aminoglycosides on the kidney, ear and balance in cystic fibrosis. Thorax 2010;65:654-8.

49. Smyth A, Lewis S, Bertenshaw C, et al. Case-control study of acute renal failure in patients with cystic fibrosis in the UK. Thorax 2008;63:532-5.

50. Goss CH. Should we stop using intravenous gentamicin in patients with cystic fibrosis? Thorax 2008:63:479-80.

51. Webb AK, Egan J. Should patients with cystic fibrosis infected with Burkholderia cepacia undergo lung transplantation? Thorax 1997;52:671-3.

52. Meachery G, De Soyza A, Nicholson A, et al. Outcomes of lung transplantation for cystic fibrosis in a large UK cohort. Thorax 2008;63:725-31.
53. De Soyza A, Meachery G, Hester KL, et al. Lung transplantation for patients with cystic fibrosis and Burkholderia cepacia complex infection: a single-center experience. J Heart Lung Transplant 2010;29:1395-404.

54. Boussaud V, Guillemain R, Grenet D, et al. Clinical outcome following lung transplantation in patients with cystic fibrosis colonised with Burkholderia cepacia complex: results from two French centres. Thorax 2008:63:732-7.

55. Murray S, Charbeneau J, Marshall BC, et al. Impact of Burkholderia infection on lung transplantation in cystic fibrosis. Am J Respir Crit Care Med 2008:178:363-71.

56. Noone PG. Lung transplant and cystic fibrosis: what's new from the UK and France? Thorax 2008;63:668-70.

57. Accurso F, Rowe S, Clancy J, et al. Effect of VX-770 in persons with cystic fibrosis and the G551D-CFTR mutation. N Engl J Med 2010;363:1991-2003.

58. Welsh MJ. Targeting the basic defect in cystic fibrosis. $N$ Eng/ J Med 2010;363:2056-7.

\section{Thorax online}

Visit Thorax online and listen to the latest podcast, post comments and download any you might have missed. Keep informed and up to date by visiting thorax.bmj.com. 